\title{
Biometría Fetal por Ultrasonidos
}

(Diámetro Biparietal)

\author{
Dr. Luis Carlos Jiménez G.* \\ Dr. Alvaro Quiroga Gil.** \\ Dr. Roberto Vergara Tamara*** \\ Dr. Humberto Mayorga**** \\ Trabajo presentado al XIII - Congreso de Obste- \\ tricia y Ginecología de Bogotá, los días 5 - 6 - 7 \\ de Diciembre de 1979.
}

\section{INTRODUCCION}

Hasta el advenimiento de los ultrasonidos y su utilización en Obstetricia, la valoración del tamaño y estado de madurez del feto intrauterino había sido acompañada de resultados muy poco fiables y de técnicas engorrosas en muchos casos.

Los ultrasonidos constituyen un método sencillo, inocuo, rápido y de una fiabilidad alta para obtener un sinnúmero de parámetros con los cuales dar respuesta a los interrogantes que se plantean referentes al crecimiento del feto $y$ a su buen o mal desarrollo intrauterino.

Utilizamos para nuestro trabajo un equipo Vidoson 635 ST, que tiene una velocidad de $1.540 \mathrm{Mts} /$ Segundo, una longitud de onda de $0,6 \mathrm{~mm}$ y una frecuencia de 2,5 Mghtz. Este trabajo presenta la primera curva del Diámetro Biparietal que se obtiene en nuestro país. Es bien conocido el concepto de que la raza, el medio socio-económico, el clima,

* Director Centro de Diagnóstico Ecográfico. Bogotá.

* Director Departamento Obstetricia, Clínica Fray Bartolomé de las Casas.

*** Director Clínica David Restrepo

**** Estadigrafo. etc., influyen en las medidas biométricas del feto, por lo cual es tan importante la obtención de ésta curva que permitirá trabajar con parámetros propios.

\section{Descripción del Universo de estudio}

A partir de agosto de 1978 se realizaron en el CENTRO DE DIAGNOSTICO ECOGRAFICO, 1.735 exploraciones ecográficas a un total de 1.127 pacientes de la CLINICA DAVID RESTREPO DE BOGOTA. Este total no incluye casos con problemas de tipo metabólico, isoinmunización, enfermedad vascular hipertensiva, enfermedad renal hipertensiva o cualquier otra patología que pueda influir de una u otra manera en los valores de las medidas fetales, distorsionando por lo tanto la calidad de los datos. Cada paciente tuvo una, dos o tres exploraciones durante su embarazo. En el cuadro No. 1 se presenta la información referente a esta situación.

Se sabe que la regularidad menstrual, es. un aspecto muy importante para la determinación de la Edad Gestacional Clínica; por esto el Universo está limitado a aquellas pacientes cuyo ciclo menstrual sea regular (cuadro No. 2).

Teniendo en cuienta las anteriores consideraciones, el universo de estudio para el presente trabajo está compuesto 
por 1.551 exploraciones ecográficas, correspondientes a 1.008 pacientes.

Cuadro No. 1

DISTRIBUCION DE LAS EXPLORACIONES ECOGRAFICAS

\begin{tabular}{|c|r|r|c|}
\hline $\begin{array}{c}\text { NUMERO DE } \\
\text { EXPLORACIONES } \\
\text { POR PACIENTE }\end{array}$ & \multicolumn{2}{|c|}{$\begin{array}{c}\text { NUMERO DE } \\
\text { PACIENTES }\end{array}$} & $\begin{array}{c}\text { TOTAL } \\
\text { EXPLORACION }\end{array}$ \\
\hline 1 & 555 & $49.24 \%$ & 555 \\
\hline 2 & 536 & $47.56 \%$ & 1.072 \\
\hline 3 & 36 & $3.20 \%$ & 108 \\
\hline Total & 1.127 & $100 \%$ & 1.735 \\
\hline
\end{tabular}

Cuadro No. 2

\section{DISTRIBUCION DEL NUMERO DE PACIENTES SEGUN SU REGULARIDAD MENSTRUAL}

\begin{tabular}{|l|c|c|}
\hline REGULARIDAD & $\begin{array}{c}\text { NUMERO } \\
\text { DE PACIENTES }\end{array}$ & PORCENTAJE \\
\hline Regulares & 1.008 & $89.44 \%$ \\
\hline Irregulares & 112 & $9.94 \%$ \\
\hline Sin dato & 7 & $0.62 \%$ \\
\hline Total & 1.127 & $100.00 \%$ \\
\hline
\end{tabular}

\section{METODOLOGIA}

La variable relevante para este primer trabajo es el Diámetro Biparietal (DBP). EI crecimiento de esta variable durante el embarazo es función principal de la edad gestacional obviamente; el interés se centró en determinar matemáticamente ecuaciones que expresen el comportamiento de dicha variable con respecto al tiempo de gestación.
Aprovechando las experiencias de otros investigadores como Bonilla, Pérez Gil, Parache, Chinchilla y por intermedio de las obras de los anteriores, de Hellman, Kratochwil, Campell, Willocks, De la Fuente, Sabbagha, Murata y Berger, se ajustaron diferentes ecuaciones de regresión usando el Método de los Mínimos Cuadrados, siendo la variable independiente la Edad Gestacional en todos los casos.

Dichas regresiones se hicieron para segundo trimestre, tercer trimestre, segundo y tercer trimestre conjuntamente $y$ en cada caso para varones, mujeres, varones y mujeres agrupadamente, con el objeto de tratar de observar alguna diferencia notable entre los dos sexos, referente al crecimiento del Diámetro Biparietal.

La forma y nornbre de las ecuaciones de regresión usadas aparecen en el cuadro No. 2A, ecuaciones que responden a ensayos hechos por los investigadores antes citados. Como puede observarse, existen otros tipos de ecuaciones pero

Cuadro No. 2A

\section{FORMA Y NOMBRE DE LAS REGRESIONES ANALIZADAS}

\begin{tabular}{|l|l|}
\hline $\begin{array}{c}\text { FORMA DE } \\
\text { LA ECUACION }\end{array}$ & NOMBRE \\
\hline$Y=a+b x$ & LINEAL \\
\hline$Y=c+d x+f x^{2}$ & PARABOLICA \\
\hline$Y=g+h l n x$ & LOGARITMICA \\
\hline$Y=i(j) x$ & EXPONENCIAL \\
\hline$Y=k x^{\prime}$ & POTENCIAL \\
\hline
\end{tabular}

\footnotetext{
$a, b, c, f, g, h, i, j, k, ~ l:$ constantes

$Y$ : variable dependiente

$X$ : variable independiente
} 
debido a que estas son las de uso corriente y además son las más simples, sólo se trabajó este conjunto de expresiones matemáticas. En cada caso se analizó el Coeficiente de Correlación simple o el Coeficiente de Correlación múltiple (en el caso de la regresión polinómica) como también otros aspectos, con el fin de seleccionar aquella ecuación que mejor se ajuste. Para tal efecto la información se proceso electrónicamente en un computador de la IBM.

\section{Resultados}

En los cuadros a continuación presentados (cuadro No. 3 al cuadro No. 5) aparecen las diferentes ecuaciones de regresión calculadas. Frente a cada expresión matemática, aparece el coeficiente de correlación lo mismo que el número de casos.

\section{Análisis de resultados}

\section{Segundo trimestre}

Realmente los coeficientes de correlación del grupo de ecuaciones calculadas para los varones, no difieren ampliamente. En efecto, el coeficiente menor es de 0.74405 presentado por la regresión rectilínea, el coeficiente mayor por la ecuación potencial el cual es de 0.79807 , es decir, que presentaron una diferencia de 0.05402 . La mejor ecuación para varones fue por lo tanto $\mathrm{DBP}=1.9892347$ (EG) 1.093722, la cual se presenta en la gráfica No. 1.

En cuanto a las regresiones hechas, para las mujeres la situación es similar, es decir, el mayor coeficiente lo presentó la ecuación potencial: 0.82620 , el menor coeficiente es el correspondiente a la ecuación logarítmica: 0.78541 , con

Cuadro No. 3

ECUACIONES DE REGRESION Y COEFICIENTES DE CORRELACION PARA EL DIAMETRO BIPARIETAL EN EL SEGUNDO TRIMESTFE

\begin{tabular}{|c|c|c|c|}
\hline \multicolumn{2}{|l|}{$\checkmark$ ARONES } & \multicolumn{2}{|l|}{ MUJERES } \\
\hline $\mathrm{DBP}=-2.247593+2.764606 \mathrm{EG}$ & $\begin{array}{c}0.74405 \\
254\end{array}$ & $\mathrm{DBP}=-9.088766+3.140678 \mathrm{EG}$ & $\begin{array}{c}0.78866 \\
186\end{array}$ \\
\hline$D B P=-26.93736+5.53164 E G-0.075599(E G)^{2}$ & $\begin{array}{c}0.74658 \\
254\end{array}$ & $\mathrm{DBP}=-6.149109+2.814303 \mathrm{EG} 0.008816615 \mathrm{EG}^{2}$ & $\begin{array}{c}0.78869 \\
186\end{array}$ \\
\hline $\mathrm{DBP}=-95.41033+49.68369 \mathrm{LN}(\mathrm{EG})$ & $\begin{array}{l}0.74652 \\
254\end{array}$ & $D B P=-115.4383+56.63533 L N(E G)$ & $\begin{array}{l}0.78541 \\
186\end{array}$ \\
\hline $\mathrm{DBP}=15.582835(1.0623171) \mathrm{EG}$ & $\begin{array}{l}0.79011 \\
254\end{array}$ & $\mathrm{DBP}=13.910932(1.0687131) \mathrm{EG}$ & $\begin{array}{l}0.82396 \\
186\end{array}$ \\
\hline$D B P=1.9892347(E G)^{1.093722}$ & $\begin{array}{c}0.79807 \\
254\end{array}$ & $\mathrm{DBP}=1.4309644\{\mathrm{EG}) 1.206595$ & $\begin{array}{l}0.82620 \\
186\end{array}$ \\
\hline
\end{tabular}

\begin{tabular}{|c|c|}
\hline \multicolumn{2}{|c|}{ VARONES Y MUJERES } \\
\hline $\mathrm{DBP}=-4.810461+2.906488 \mathrm{EG}$ & $\begin{array}{c}0.76296 \\
440\end{array}$ \\
\hline $\mathrm{DBP}=-11.53323+3.657602 \mathrm{EG}-0.02042412$ (EG) 2 & $\begin{array}{c}0.76313 \\
440\end{array}$ \\
\hline $\mathrm{DBP}=-102.5425+52.16909$ LN (EG) & $\begin{array}{c}0.76159 \\
440\end{array}$ \\
\hline $\mathrm{DBP}=15.003087(1.0644605) \mathrm{EG}$ & $\begin{array}{c}0.80322 \\
440\end{array}$ \\
\hline $\mathrm{DBP}=1.7944335$ (EG) 1.129158 & $\begin{array}{c}0.80744 \\
440\end{array}$ \\
\hline
\end{tabular}


Cuadro No. 4

ECUACIONES DE REGRESION Y COEFICIENTES DE CORRELACION PARA EL DIAMETRO DIPARIETAL EN EL TERCER TRIMESTRE

\begin{tabular}{|c|c|c|c|}
\hline \multicolumn{2}{|l|}{ VARONES } & \multicolumn{2}{|l|}{ MUJERES } \\
\hline$D B P=15.53471+2.180603 E G$ & $\begin{array}{l}0.81096 \\
493\end{array}$ & $\mathrm{DBP}=12.78146+2.232182 \mathrm{EG}$ & $\begin{array}{c}0.77342 \\
431\end{array}$ \\
\hline$D B P=-77.09649+8.264759 E G-0.0986313 E G^{2}$ & $\begin{array}{c}0.82248 \\
493\end{array}$ & $D B P=-78.37038+8.228968 E G-0.09743688(E G)^{2}$ & $\begin{array}{l}0.78245 \\
431\end{array}$ \\
\hline$D B P=-146.7086+67.05676 L N(E G)$ & $\begin{array}{c}0.81734 \\
493\end{array}$ & $D B P=-152.8285+68.49851$ LN (EG) & $\begin{array}{l}0.77903 \\
431\end{array}$ \\
\hline $\mathrm{DBP}=35.458784(1.0276271) \mathrm{EG}$ & $\begin{array}{l}0.76869 \\
493\end{array}$ & $\mathrm{DBP}=33.714769(1.028073) \mathrm{EG}$ & $\begin{array}{c}0.72965 \\
431\end{array}$ \\
\hline$D B P=4.6432474(E G) 0.8395948$ & $\begin{array}{c}0.77616 \\
493\end{array}$ & $D B P=4.0699674(E G) 0.8736182$ & $\begin{array}{c}0.73672 \\
431\end{array}$ \\
\hline
\end{tabular}

\begin{tabular}{|c|c|}
\hline \multicolumn{2}{|c|}{ VARONES Y MUJERES } \\
\hline DBP $=14.43055+2.199429 E \mathrm{E}$ & $\begin{array}{c}0.79249 \\
824\end{array}$ \\
\hline $\mathrm{DBP}-77.330625+8.230588 \mathrm{EG}-0.09789375(\mathrm{EG}) 2$ & $\begin{array}{c}0.80279 \\
824\end{array}$ \\
\hline $\mathrm{DBP}-148.952+67.55632 \mathrm{LN}(\mathrm{EG})$ & $\begin{array}{c}0.79849 \\
824\end{array}$ \\
\hline $\mathrm{DBP}=34.73254(10280929) \mathrm{EG}$ & $\begin{array}{c}0.74931 \\
824\end{array}$ \\
\hline $\mathrm{DBP}=4.4078983$ (EG) 0.8527921 & $\begin{array}{c}0.75659 \\
824\end{array}$ \\
\hline
\end{tabular}

una diferencia de 0.04079 un poco menor que para el caso de los varones. Es por esto que la mejor ecuación es para este caso DBP $=1.4309644(E G)$ 1.206595 que puede observarse en el gráfico No. 1.

La mejor ecuación cuando se consideran conjuntamente varones y mujeres fue $\mathrm{DBP}=1.7944335(\mathrm{EG}) 1.12918$ con un coeficiente de correlación de 0.80744 mostrada en la gráfica No. 1 .

\section{Tercer trimestre}

Como cambio notable en cuanto al mejor ajuste, pudo observarse que el ajuste potencial en este trimestre ocupó el último lugar, pues tanto para fetos varones como para fetos mujeres, lo mismo que para el caso en que se consideran conjuntamente, fue la que presentó más bajo coeficiente. Las mejores ecuaciones fueron del tipo parabólico así:

\section{Para varones}

$\mathrm{DBP}=$

$-77.096498 .264759(E G)-0.0986314(E G E)^{2}$

Para mujeres

$\mathrm{DBP}=$

$-78.370388 .228968(E G)-0.0974368(E G)^{2}$

Para varones y mujeres

DBP :

$-77.3306258 .230588(E G)-0.09789375(E G)^{2}$

Los coeficientes de correlación fueron: $0.82248,0.78245$ y 0.80279 , respectivamente. 
GRAFICA No. 1

CRECIMIENTO DEL DIAMETRO BIPARIETAL

SEGUNDO TRIMESTRE

HOMBRES Y MUJERES

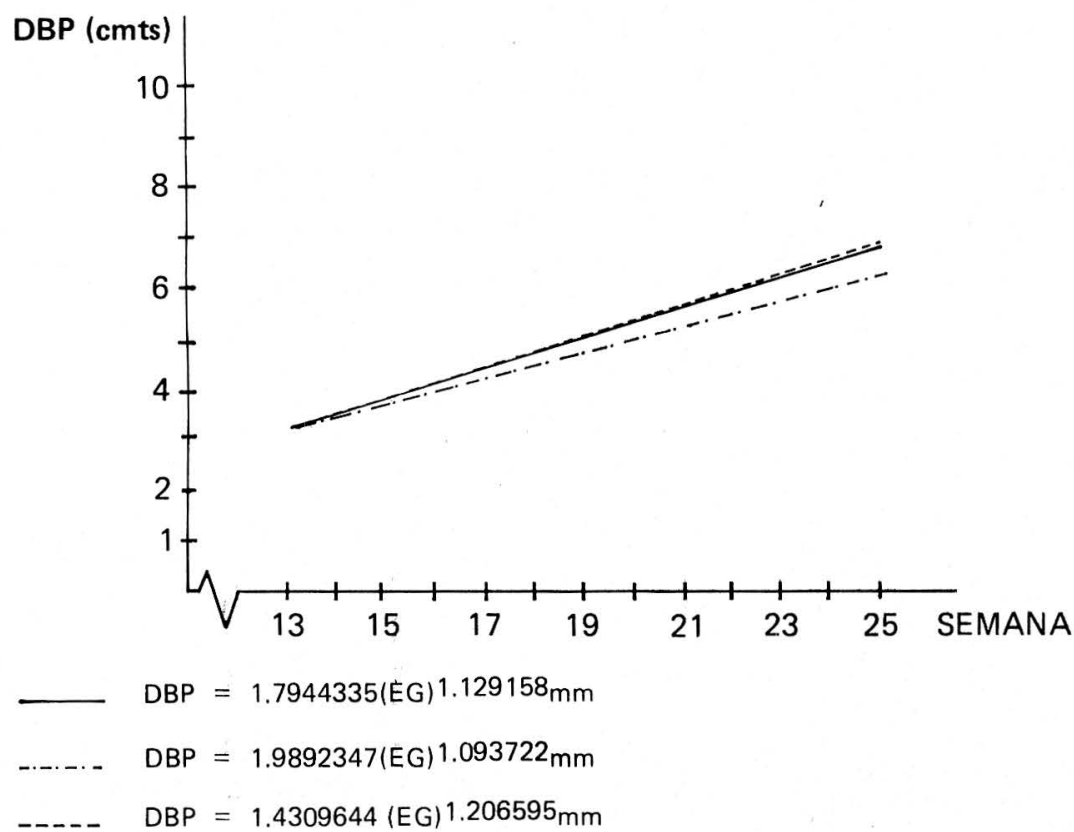

Las anteriores ecuaciones son presentadas en la gráfica No. 2 .

Otro aspecto que se notó fue el siguiente: un cambio en la pendiente de la regresión rectilínea del segundo trimestre al tercer trimestre. Para los varones pasa de 2.764606 a 2.180603 , en el caso de las mujeres pasa de 3.140678 a 2.232182 y en el caso en el que se consideran conjuntamente pasa de 2.906484 a 2.199429.

En la curva exponencial se observó una situación similar. La rata de crecimiento compuesto pasa del $6.23171 \%$ al $2.76271 \%$ para el caso de los fetos varones, de $6.87131 \%$ al $2.8073 \%$ en los fetos mujeres y del $6.44605 \%$ al $2.80929 \%$ cuando se consideró conjuntamente los dos sexos. Igualmente, en la curva potencial, la potencia desciende en todos los casos así: de 1.206595 a 0.8736182 considerando solamente mujeres de 1.0937 a 0.8395 cuando se consideró solamente varones y de 1.129158 a 0.8527921 considerando conjuntamente varones y mujeres.

Esto indudablemente nos hace concluir que el crecimiento del Diámetro Biparietal es más rápido en el segundo trimestre que en el tercerc. 
Cuadro No. 5

ECUACIONES DE REGRESION Y COEFICIENTES DE CORRELACION PARA EL DIAMETRO BIPARIETAL EN EL SEGUNDO Y TERCER TRIMESTRE

\begin{tabular}{|c|c|c|c|}
\hline \multicolumn{2}{|l|}{ VARONES } & \multicolumn{2}{|l|}{ MUJERES } \\
\hline$D B P=-0.4299339+2.664241 E G$ & $\underset{744}{0.94423}$ & DBP $\quad 1.194653+2.593496$ EG & $\begin{array}{c}0.92917 \\
615\end{array}$ \\
\hline $\mathrm{DBP}-22.44938+4.515421 \mathrm{EG}-0.03607437(\mathrm{EG})^{2}$ & $\begin{array}{l}0.94737 \\
744\end{array}$ & $\mathrm{OBP}=-22.55275+4.571822 \mathrm{EG}-0.03839854 \mathrm{EG}^{2}$ & $\begin{array}{c}0.93290 \\
615\end{array}$ \\
\hline$D B P=-139.5289+64.7471$ LN (EG) & $\begin{array}{c}0.94539 \\
744\end{array}$ & $D B P=-135.7661+63.46977$ LN (EG) & $\begin{array}{c}0.93186 \\
615\end{array}$ \\
\hline$D B P=21.909734(1.043103) E G$ & $\begin{array}{c}0.92708 \\
744\end{array}$ & $D B P=22.412216(1.0421597) E G$ & $\begin{array}{c}0.91279 \\
615\end{array}$ \\
\hline$D B P=2.2952221(E G) 1.041744$ & $\begin{array}{c}0.94287 \\
744\end{array}$ & $\mathrm{DBP}=2.4001245(\mathrm{EG}) 1.026839$ & $\begin{array}{l}0.93014 \\
619\end{array}$ \\
\hline$D B P=110 / 1+e^{2.334099}-0.1124769 E G$ & $\begin{array}{l}0.94472 \\
744\end{array}$ & $D B P=110 / 1+e^{2.261034}-0.1089724 E G$ & $\frac{0.92797}{615}$ \\
\hline
\end{tabular}

\begin{tabular}{|c|c|}
\hline \multicolumn{2}{|l|}{ VARONES Y MUJERES } \\
\hline $\mathrm{DBP}=0.2943039+2.632672 \mathrm{EG}$ & $\begin{array}{r}0.93799 \\
1.382\end{array}$ \\
\hline $\mathrm{DBP}=-22.08053+4.50765 \mathrm{EG}-0.036499(\mathrm{EG})^{2}$ & $\begin{array}{l}0.94129 \\
1.382\end{array}$ \\
\hline OBP $=-137,6128+64,1039$ LN (EG) & $\begin{array}{c}0.93961 \\
1.382\end{array}$ \\
\hline$D B P=22.134711(1.0426832) E G$ & $\begin{array}{c}0.92124 \\
1.382\end{array}$ \\
\hline$D B P=2.3515319(E G) 1.033817$ & $\begin{array}{c}0.93741 \\
1.382\end{array}$ \\
\hline 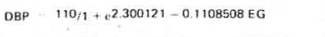 & $\begin{array}{l}0.93768 \\
1.382\end{array}$ \\
\hline
\end{tabular}

\section{GRAFICA No. 2}

CRECIMIENTO DEL DIAMETRO BIPARIETAL

TERCER TRIMESTRE

HOMBRES Y MUJERES

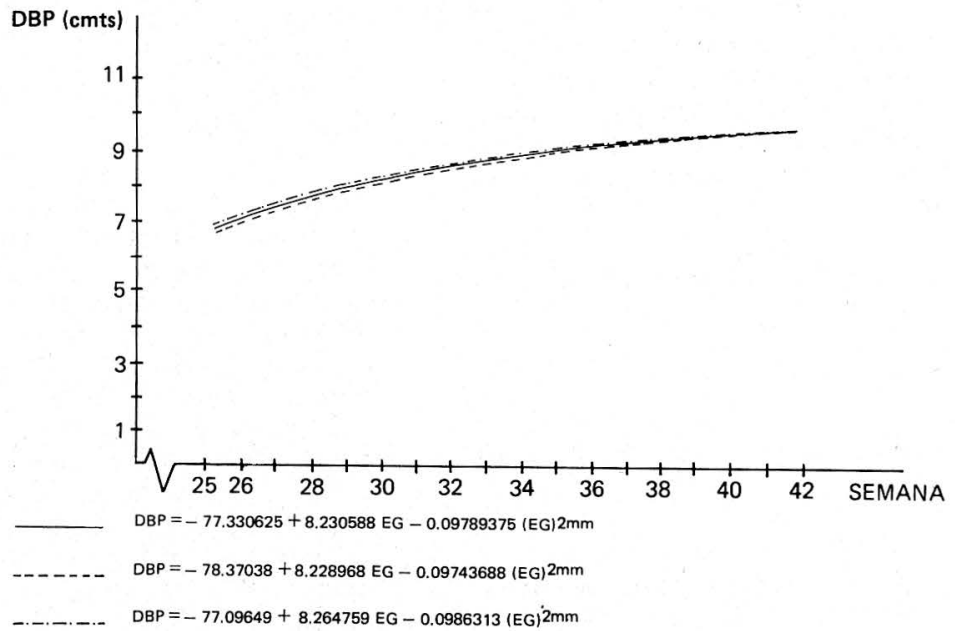




\section{Segundo y tercer trimestre}

Como puede verse en el cuadro No. 5 , las mejores ecuaciones de regresión fueron:

\section{Para varones}

$\mathrm{DBP}=-22.449384 .515421(\mathrm{EG})-0.360743(\mathrm{EG})^{2}$, con coeficiente de 0.94737 .

Para mujeres

$\mathrm{DBP}=-22.552754 .571822(\mathrm{EG})-0.03839854(E G)^{2}$ con coeficiente de 0.93290 .

Para varones y mujeres conjuntamente $\mathrm{DBP}=22.080534 .50765(E G)-0.36499(E G)^{2}$. con coeficiente de 0.94129 .

Dichas curvas pueden apreciarse en la gráfica No. 3.

En base al análisis que se hizo en 5.2 en cuanto al cambio en la rapidez del crecimiento del Diámetro Biparietal del segundo trimestre al tercero y analizando los diferentes tipos de ecuaciones creemos que el crecimiento del Diámetro Biparietal es aproximadamente de tipo Logístico lo cual obviamente puede ponerse a prueba en investigaciones similares para evaluar su confiabilidad debido a que como se está describiendo el comportamiento en el universo anteriormente descrito, es imposible cualquier generalización debido a las características particulares que posee dicho universo.

Una curva Logística es de la forma $Y=\frac{k}{1+e^{a+b x}} \quad$ la cual

gráficamente puede verse en el gráfico No. 4.

El valor de $k$ es una asíntota de la gráfica, es decir, aquel valor al cual la gráfica se va acercando cuando se toman valores grandes de $X$, el valor es una constante que es la base de los logaritmos Neperianos y cuyo valor es aproximadamente 2.7182818 , los valores $a$ y b son obtenidos a partir de los datos usando el método de los Mínimos Cuadrados y una transformación especial.

El valor de la variable $X$ donde la curva cambia su convexidad, es el punto $X=\frac{-a}{b}$ y se llama punto $d e$ inflexión.

Para el caso la curva que se pone a consideración y que aparece en el gráfico No. 5 es:

$$
D B P=\frac{110}{1+e^{2.300121-0.1108508 E G}}
$$

esta curva presenta un coeficiente de 0.93768 que aunque no es el coeficiente más alto, si expresa la forma como cambia el crecimiento del Diámetro Biparietal en los últimos trimestres de embarazo. El punto de inflexión de la curva teniendo en cuenta que $a=2.300121 \mathrm{y}$ $\mathrm{b}=-0.1108508$ es aproximadamente 21 (20.749701). Esto indica que a partir de la semana 21 el Diámetro Biparietal crece más lentamente que en las semanas anteriores.

En cuanto al índice de crecimiento del Diámetro Biparietal a partir de los resultados arrojados por las ecuaciones y por facilidad, tomando la rectil ínea se puede decir, que aproximadamente los varones crecen durante el segundo trimestre 2.76 milímetros por semana y $2.18 \mathrm{mi}$ límetros cada semana en el tercer trimestre, las mujeres crecen 3.14 milímetros por semana durante el segundo trimestre y 2.23 milímetros por semana en el tercer trimestre. Conjuntamente crecen 2.9 y 2.19 milímetros por semana durante el segundo $y$ tercer trimestre respectivamente. 
GRAFICA No. 3

CRECIMIENTO DEL DIAMETRO BIPARIETAL

SEGUNDO Y TERCER TRIMESTRE

HOMBRES Y MUJERES

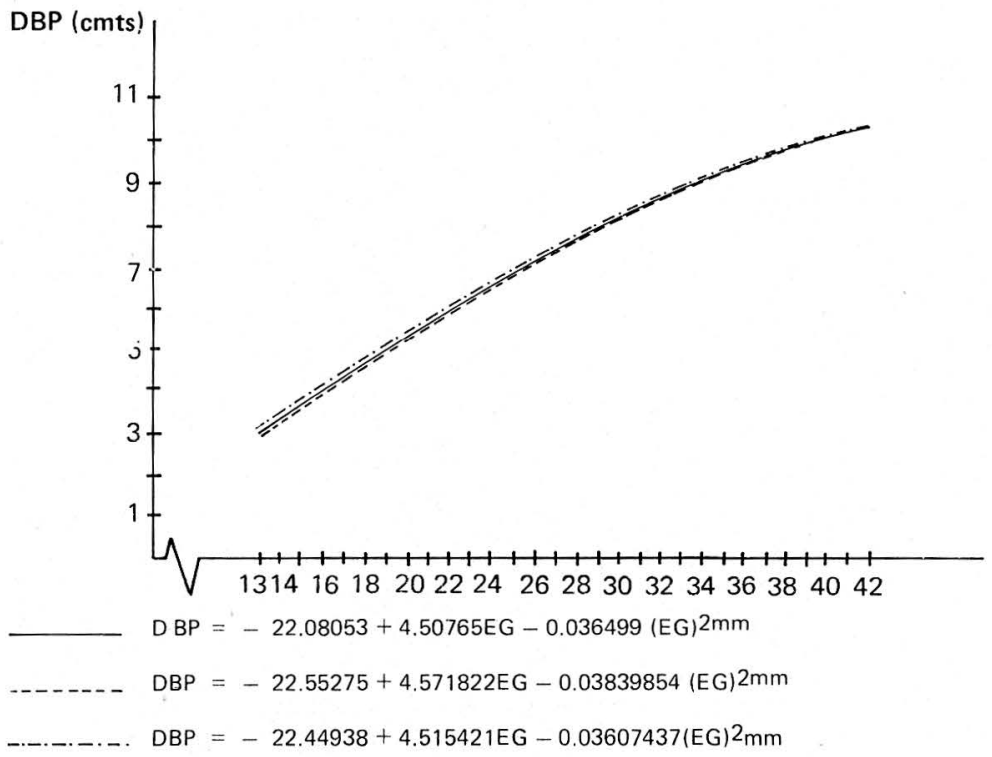

GRAFICA No. 4

UNA CURVA DE TIPO LOGISTICO

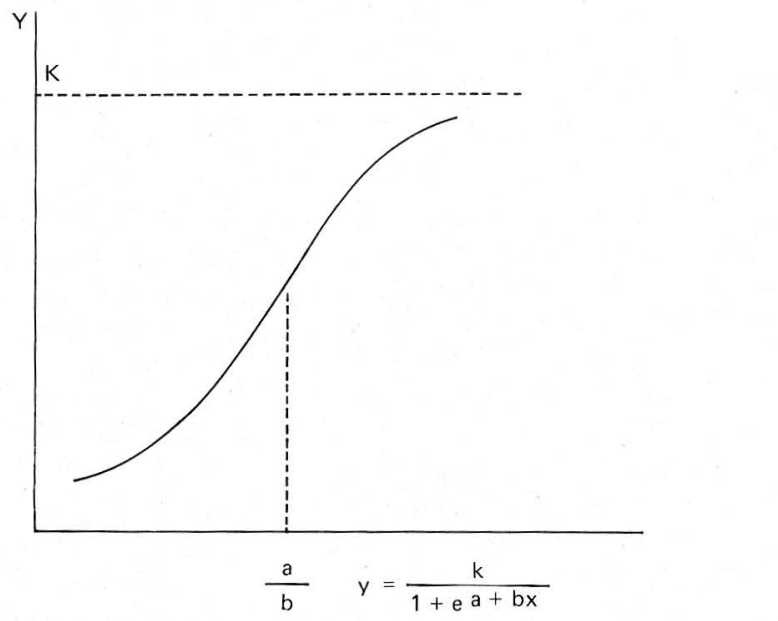


GRAFICA No. 5

\section{CRECIMIENTO DEL DIAMETRO BIPARIETAL}

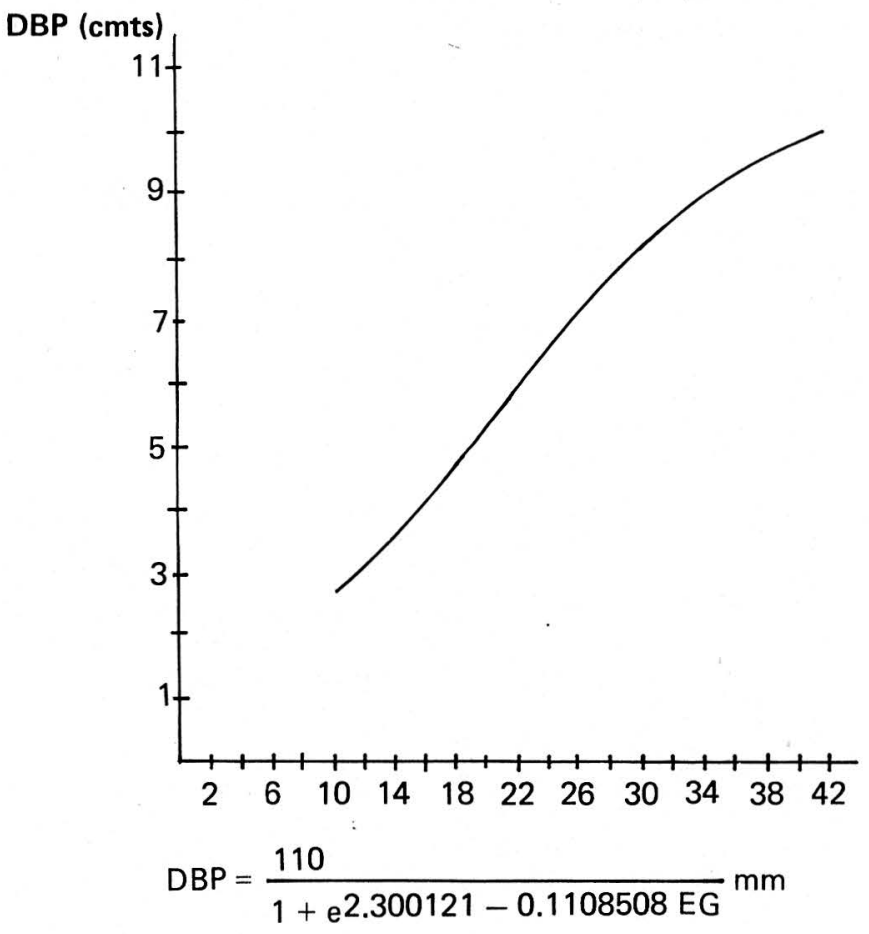

\section{ULTRASONIC FETAL BIOMETRY}

\section{Summary}

We applied to a total of 1.551 echographic explorations different retrogression curves per quarter and per fetus sex in order to find the best formula which can express the D.B.P.'s growth with respect to the gestational age.

During these tests it was possible to determine the growth differences between the second and the third quarter. The best curve chosen was the logistic one since it gave a better description of such differences and showed the best coefficient.

\section{Conclusiones}

6.1. La diferencia de crecimiento del D.B.P. en cuanto al sexo se refiere no es estadísticamente significativa.

6.2. El crecimiento del D.B.P. presenta diferencias entre el segundo y tercer trimestre como se destaca en 2.

6.3. Se escoge la curva de crecimiento de tipo logistico del D.B.P. porque presenta un buen coeficiente $y$ además describe la diferencia de 
Cuadro No. 6

ESTIMACIONES POR MEDIO DE DIFERENTES ECUACIONES DE DIAMETRO BIPARIETAL EN EL SEGUNDO $Y$ TERCER SEMESTRE

\begin{tabular}{|c|c|c|c|c|c|c|}
\hline semana & recta & parábola & logarítmica & exponencial & potencial & logística \\
\hline 12 & 3.19 & 2.67 & 2.17 & 3.65 & 3.14 & 3.02 \\
\hline 13 & 3.45 & 3.03 & 2.68 & 3.81 & 3.41 & 3.27 \\
\hline 14 & 3.71 & 3.39 & 3.16 & 3.97 & 3.68 & 3.53 \\
\hline 15 & 3.98 & 3.73 & 3.60 & 4.14 & 3.96 & 3.80 \\
\hline 16 & 4.24 & 4.07 & 4.01 & 4.32 & 4.24 & 4.08 \\
\hline 17 & 4.50 & 4.40 & 4.40 & 4.50 & 4.51 & 4.37 \\
\hline 18 & 4.77 & 4.72 & 4.77 & 4.70 & 4.79 & 4.67 \\
\hline 19 & 5.03 & 5.04 & 5.11 & 4.90 & 5.07 & 4.97 \\
\hline 20 & 5.30 & 5.35 & 5.44 & 5.11 & 5.34 & 5.27 \\
\hline 21 & 5.60 & 5.65 & 5.75 & 5.32 & 5.62 & 5.58 \\
\hline 22 & 5.82 & 5.94 & 6.05 & 5.55 & 5.90 & 5.88 \\
\hline 23 & 6.08 & 6.23 & 6.34 & 5.79 & 6.18 & 6.18 \\
\hline 24 & 6.35 & 6.51 & 6.61 & 6.03 & 6.46 & 6.48 \\
\hline 25 & 6.61 & 6.78 & 6.87 & 6.30 & 6.74 & 6.77 \\
\hline 26 & 6.87 & 7.04 & 7.12 & 6.56 & 7.03 & 7.06 \\
\hline 27 & 7.14 & 7.30 & 7.34 & 6.84 & 7.31 & 7.33 \\
\hline 28 & 7.40 & 7.55 & 7.60 & 7.13 & 7.59 & 7.60 \\
\hline 29 & 7.66 & 7.79 & 7.82 & 7.44 & 7.87 & 7.85 \\
\hline 30 & 7.93 & 8.03 & 8.04 & 7.76 & 8.16 & 8.09 \\
\hline 31 & 8.19 & 8.26 & 8.25 & 8.09 & 8.44 & 8.33 \\
\hline 32 & 8.45 & 8.48 & 8.46 & 8.43 & 8.72 & 8.54 \\
\hline 33 & 8.71 & 8.69 & 8.65 & 8.80 & 9.01 & 8.75 \\
\hline 34 & 8.98 & 8.89 & 8.84 & 9.17 & 9.29 & 8.94 \\
\hline 35 & 9.24 & 9.10 & 9.03 & 9.56 & 9.58 & 9.12 \\
\hline 36 & 9.50 & 9.29 & 9.21 & 9.97 & 9.86 & 9.30 \\
\hline 37 & 9.77 & 9.47 & 9.40 & 10.39 & 10.15 & 9.44 \\
\hline 38 & 10.03 & 9.65 & 9.60 & 10.83 & 10.44 & 9.58 \\
\hline 39 & 10.30 & 9.82 & 9.72 & 11.30 & 10.72 & 9.72 \\
\hline 40 & 10.60 & 9.98 & 9.89 & 11.78 & 11.01 & 9.84 \\
\hline 41 & 10.82 & 10.14 & 10.00 & 12.28 & 11.30 & 9.95 \\
\hline 42 & 11.08 & 10.29 & 10.20 & 12.80 & 11.58 & 10.05 \\
\hline
\end{tabular}

crecimiento en los dos últimos trimestres.

\section{Resumen}

A nuestro universo de 1.551 explora- ciones ecográficas, aplicamos diferentes curvas de regresión por trimestre y por sexo del feto para encontrar la mejor ecuación que exprese el crecimiento del D.B.P. en función de la edad gestacional 
A lo largo de estos ensayos se pudo establecer las diferencias de crecimiento entre el segundo y tercer trimestre; escogiéndose como la mejor curva la de tipo logístico por describir mejor dichas diferencias y presentar el mejor coeficiente.

\section{Bibliografía}

1. BONILLA MUSOLES. Diagnóstico por ultrasonido en Obstetricia y Ginecología.
2. BERNARD OSTLE. Estadística Aplicada.

3. GRAYBILL FRANKLIN A. An Introduction to linear statistical models. Vol. 1.

4. PARACHE JAVIER. Ecografía Bidimensional en Obstetricia.

5. SEARLE S. R. Linear Models. 